\title{
Purpose and procedure
}

The general purpose of Evidence-Based Medicine is to select from the health-related literature* those articles reporting important advances in internal medicine, general and family practice, surgery, psychiatry, paediatrics, and obstetrics and gynaecology, and whose results are most likely to be both true and useful. These articles are described, critiqued and commented on by clinical experts. The specific purposes of Evidence-Based Medicine are:

- to identify, using predefined criteria, the best original and review articles on the cause, course, diagnosis, prevention, treatment, quality of care, or economics of disorders in the foregoing fields

- to provide a description and expert commentary on the context of each article, its methods, and the clinical applications that its findings warrant

- to disseminate the summaries in a timely fashion

The BMJ Publishing Group publishes Evidence-Based Medicine. A new editorial board is currently being assembled; articles for the first two issues of 2010 are being chosen by the content editors, Bazian Ltd, using the selection criteria defined below.

\section{Criteria for selection and review of articles for abstracting}

All articles in a journal issue are considered for inclusion if, based on their abstracts, they meet the following basic and category-specific criteria:

\section{Basic criteria}

All English-language original and review articles in an issue of a candidate journal are considered for abstracting if they concern topics important to the clinical practice of internal medicine, general and family practice, surgery, psychiatry, paediatrics, or obstetrics and gynaecology

\section{Category-specific criteria}

Prevention of treatment, quality improvement

- Random allocation of participants to interventions

- Outcome measures of known or probable clinical importance

\section{Diagnosis}

- Inclusion of a spectrum of participants, some (but not all) of whom have the disorder or derangement of interest

- Each participant must receive the new test and the diagnostic standard test

- Either an objective diagnostic standard or a contemporary clinical diagnostic standard with demonstrably reproducible criteria for any subjectively interpreted component

- Interpretation of the test without knowledge of the diagnostic standard result

- Interpretation of the diagnostic standard without knowledge of the test result

\section{Prognosis}

- An inception cohort of persons, all initially free of the outcome of interest

- Follow-up of $>80 \%$ of patients until the occurrence of either a major study end point or the end of the study

\section{Causation}

- Observations concerning the relation between exposures and putative clinical outcomes

- Prospective data collection with clearly identified comparison group(s) for those at risk for the outcome of interest (in descending order of preference from randomised controlled trials, quasi-randomised controlled trials, nonrandomised controlled trials, cohort studies with case by case matching or statistical adjustment to create comparable groups, to nested case-control studies)

- Masking of observers of outcomes to exposures (this criterion is assumed to be met if the outcome is objective)

\section{Economics of healthcare programmes or interventions}

- The economic question must compare alternative courses of action in real or hypothetical patients

- The alternative diagnostic or therapeutic services or quality improvement strategies must be compared on the basis of both the outcomes they produce (effectiveness) and the resources they consume (costs)

- Evidence of effectiveness must come from a study (or studies) that meets criteria for diagnosis, treatment, quality assurance, or review articles

- Results should be presented in terms of the incremental or additional costs and outcomes incurred and a sensitivity analysis should be done

\section{Clinical prediction guides}

- The guide must be generated in 1 set of patients (training set) and validated in an independent set of real not hypothetical patients (test set), and must pertain to treatment, diagnosis, prognosis, or causation

\section{Differential diagnosis}

- A cohort of patients who present with a similar, initially undiagnosed but reproducibly defined clinical problem

- Clinical setting is explicitly described

- Ascertainment of diagnosis for $>80 \%$ of patients using a reproducible diagnostic workup strategy and follow-up until patients are diagnosed or follow-up of $>1$ month for acute disorders or $>1$ year for chronic or relapsing disorders

\section{Systematic reviews}

- The clinical topic being reviewed must be clearly stated; there must be a description of how the evidence on this topic was tracked down, from what sources, and with what inclusion and exclusion criteria

- $>1$ article included in the review must meet the above-noted criteria for treatment, diagnosis, prognosis, causation, quality improvement, or the economics of healthcare programmes

Articles meeting the criteria set out above and chosen for coverage in Evidence-Based Medicine are described and reviewed by an expert in the content area covered by the article. This expert writes a commentary in which she or he describes the article and compares the study findings to previous research findings, identifies any important methodological problems that affect interpretation of the study results, and offers recommendations for clinical application.

\section{* Journals currently reviewed}

For the first two issues of 2010 the journals being covered are those of the Core Clinical (Abridged Index Medicus) Journal collection in PubMed Medline. Searches were carried out in the Core collection using the following search strategy:

1 diagnos* OR prevalence OR etiology OR aetiology OR random* OR meta-analysis OR Systematic review OR (Systematic AND review)

2 cochrane [journal name]

31 OR 2

Table of Contents screening for select key clinical journals will be reintroduced for the third issue of 2010. 